\title{
Persuasive Health and Wellbeing Application: A Theory-Driven Design in Promoting Physical Activity
}

\author{
Md Sanaul Haque ${ }^{1,6}$, Wali Mohammad Abdullah ${ }^{2,7}$, Sadiqur Rahaman ${ }^{3,8}$, Maarit Kangas ${ }^{4,6}$ and Timo Jämsä ${ }^{4,5,6}$ \\ ${ }^{1}$ Information Processing Science, University of Oulu, Oulu, Finland \\ ${ }^{2}$ Computer Science and Engineering, Military Institute of Science and Technology, Dhaka, Bangladesh \\ ${ }^{3}$ Center for Wireless Communication, University of Oulu, Oulu, Finland \\ ${ }^{4}$ Research Unit of Medical Imaging, Physics and Technology, University of Oulu, Oulu, Finland \\ ${ }^{5}$ Medical Research Center Oulu, Oulu University Hospital and University of Oulu, Oulu, Finland \\ Email: ${ }^{6}\left\{\right.$ md.haque, maarit.kangas, timo.jamsa\}@oulu.fi, ${ }^{7}$ shashi.mist@gmail.com, ${ }^{8}$ srahaman@ee.oulu.fi
}

\begin{abstract}
People spend a lot of time at their workplace, for example in office environment. After consuming breakfast or lunch during the recess periods, one may feel lazy both physically and mentally. Thus, gaining extra calories may lower the working progress. To motivate individuals for healthier behavior, we designed, executed and tested an application for improving physical activity behavior at workplace. Our health and wellbeing application delivers a physical activity promotion intervention that motivates user performing activities, for example by walking after breakfast or lunch. Our physical activity promotion is based on recent research showing that one can be motivated extrinsically through satisfaction of three basic psychological needs (autonomy, competence and relatedness) that are influenced by autonomy support. We conducted a one week long usability evaluation on users for the health and wellbeing application. We found that the application works well for physical activity promotion.
\end{abstract}

Keywords-physical activity; health promotion; motivation; theory.

\section{INTRODUCTION}

Regular physical activities are useful for health, mental and physical comfort [1]. The World Health Organization (WHO) stated that 60 to $85 \%$ of adults worldwide are leading inactive lifestyles [2]. It is recommended for every adult to participate in 150 minutes of physical activity per week [3]. Past data have indicated that $60 \%$ of adults in Europe were not engaged in any physical exercise on a typical week [4]. In US, more than $50 \%$ of adults are physically not active [5]. Physical inactivity is related to the change of typical physiologic processes resulting to reduced exercise capacity, muscle atrophy, and altered energy balance etc. [6]. Physical inactivity leads to the risk of obesity, stroke, type 2 diabetes and mental health problems. Various chronic diseases are caused by these risk factors that lead causes of death [7]. On the other hand, regular physical activity prevents modern society diseases e.g. heart diseases, diabetes, depression and cancer [8][9][10]. Physical activity is beneficial to weight management as well as to an individual health i.e. selfregulating weight loss. However, workplace has been recognized by the $\mathrm{WHO}$ as a priority setting in promoting health [11]. An organization could be befitted by physical activity as its active workforce tend to experience less work absence and report less illness, work satisfaction and feel more productive. It is now a need to promote health by performing some physical activities during working hours.

Technology can be used to support behavior change for example physical activity through persuasion. Two primary ways, external behavior and internal behavior, influence the techniques of persuasion. Most persuasive applications concentrate on external behavior [12], for example a tracker for exercise [13][14]. External behaviors are more available tangible and hence, convenient for influencing. In recent times, persuasive applications have been designed for psychological influence, for example displaying virtual garden to inspire emotive linking to personal level of physical activity in the context of health and wellbeing [14][15]. Therefore, promotion of physical activities through health and wellbeing application is prospective, supporting us e.g. to provide a reminder about to do some exercises and keeping those records for judging oneself about the health condition. Two types of behavioral change based health applications are available: educational and motivational [16]. Research has shown that physical activities increase work productivity [17]. Our study concentrated on how to motivate individuals to boost their physical activity at workplace, for example office environment. Moreover, to initiate and maintain behavioral change, i.e. physical activity behavioral change, multiple health apps are required for people [16]. Past research has suggested evaluating the effectiveness of health and wellbeing applications in promoting physical activity [18].

Five key physical activities behavioral change techniques are available on most of the health applications, providing feedback on performance, prompt self-monitoring on behavior, specific goal setting, and social change, providing contingent rewards and providing instructions [18]. Studies wherein behavior change theories in applications were applied have determined that applications generally lack the practice of theoretical concepts [19][20][21]. This leads to the question: Can we integrate psychological construct in 
designing health and wellbeing application and can this application change physical activity behavior at workplace? Based on the literature reviewing for established theories, selfdetermination theory (SDT) was selected for this approach in the study. Self-determination theory has some advantage to control individual's behavior to make him/her encouraged by three basic psychological needs known as autonomy, competence and relatedness. We introduced a persuasive health and wellbeing application named iGO by using these psychological needs influenced by autonomy support to motivate people at office environments.

This paper is categorized in five sections. Section I presents introduction. In the Section II, theoretical background (SDT) is presented. Section III presents system design of the application iGO integrating SDT. Section IV presents the evaluation, i.e. post-questionnaires and survey prepared from the users of iGO and directions in furthering research work. Lastly, Section V completes the paper.

\section{THEORETICAL BACKGROUND}

SDT [22] has been appreciated in the health science domain particularly in the physical activity research [23]. In SDT, physical activity behavioral change process is described by autonomy support, psychological needs and motivation [22]. Autonomy support means individual's view of his/her social atmosphere that facilitates options and choices, accepts individual's opinion and justification when suggesting selections. Individual's psychological needs meant for autonomy, competence and relatedness are influenced positively by higher level of autonomy support. In the physical activity, autonomy can be derived as "the perceived origin or source of one's own behavior" [22]. Competence can be derived as - "feeling effective in one's ongoing interactions with the social environment and experiencing opportunities to exercise and express one's capabilities" [22]. Relatedness is the urge to feel connected to others [24]. Hence, greater level of self-determined motivation is the result of the three psychological needs. In SDT, the three types of motivation intrinsic, amotivation and extrinsic are found. Intrinsic motivation implies for doing something as it is intrinsically enjoyable or interesting. Amotivation refers to absence of willingness to participate in physical activity. On extrinsic motivation, one tends to do activity because of receiving rewards/benefits at the end of the activity. Self-determination motivation with higher level is related to more physical activity involvement [25][26]. In physical activity context, SDT has been used, but not in the entire SDT sequence (autonomy support - psychological needs - self-determination motivation). The efficacy of SDT in the context of physical activity has been tested recently [27] but not particularly at the workplace. In our case, SDT is studied at the workplace context to motivate the working people/employee after breakfast and lunch, resulting to positive working hour productivity.

However, SDT theory model of health behavioral change has been introduced recently [28]. As depicted, one's experience of autonomy, competence and relatedness are affected by health maintaining environment [29] and according to the model, satisfaction of autonomy, competence and relatedness leads to positive outcomes for mental health i.e. higher quality of life, less anxiety, depression and somatization and physical health i.e. non-smoking, workout/exercise, weight loss, glycemic control, medication use, healthier diet and dental hygiene). In our case, we are promoting the physical health outcomes, e.g. exercise/walking and weight control.
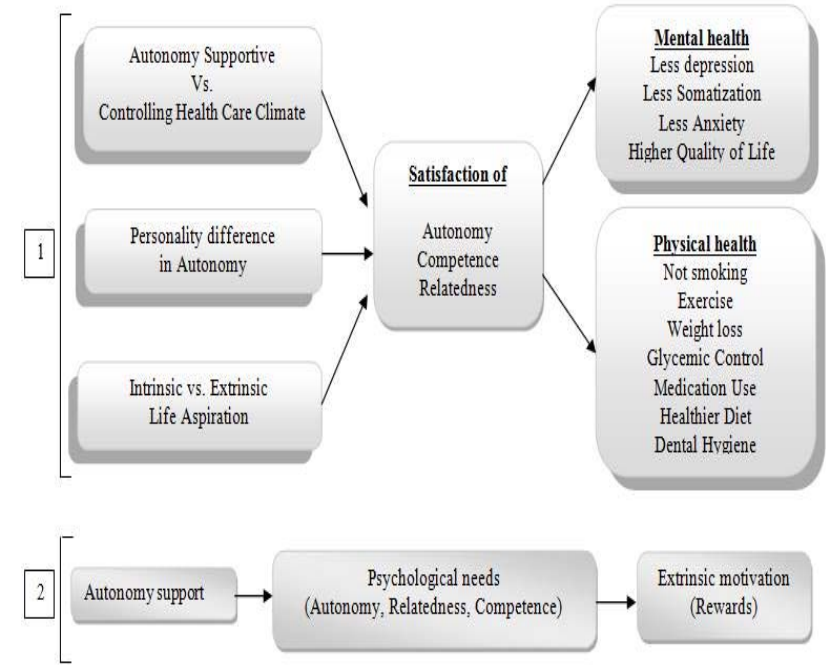

Fig. 1. Ryan's SDT theory model of health behavioral change (1) and our approach of entire SDT sequence (2) (autonomy support psychological needs - self-determination motivation)

Figure 1 represents Ryan's SDT theory model of health behavioral change [30] marked by (1) and our approach in promoting physical activity behavior (autonomy support satisfaction of psychological needs: autonomy, relatedness and competence - extrinsic motivation: rewards) marked by (2). Although the above outcomes on physical activity are promising, they measured only exercise/walking/weight loss in a generic context and physical activities at workplace were not assessed. Our study will be the first in promoting the physical health outcomes particularly physical activity e.g. exercise/walking/weight control at workplace.

A previous study discovered that SDT could be particularly beneficial for understanding physical activity motivation [31]. SDT holds significant call as an approach to realize both persistence and initiation issues in physical activity. Thus, SDT theory stated the function of motivation to boosting motivational improvement and wellbeing [32]. SDT gives an extensive flexibility to realize the patterns of physical activity behavior, wellbeing results in relation to physical activity e.g. walking/exercise, basic psychological needs and autonomy supportive factors that promotes motive for physical activity.

Recently, in Canada, a toolkit for workplaces has been facilitated for promoting physical activity at the workplace that considers exercise breaks, e.g. stretching, exercising hands and wrists, office yoga etc. [33]. However, this does not integrate any technological tools or any psychological constructs. People might forget doing exercise breaks regularly. A mobile application was developed to lessen screen-time and encourage physical activity in adolescent boys 
who were at peril of obesity [34]. Although participants were satisfied, the application was not used extensively. Another study showed that physical activity, for example walking, after a meal is more functioning for weight loss [35]. Therefore, by doing physical activity at the workplace after meal (breakfast/lunch), one may be able to control his/her weight-as well as feel less ill, more satisfaction and more productive at work. Our study is concentrated to design an application to promote physical activity particularly at the workplace i.e. after breakfast/lunch time. To the extent of our knowledge, little is known about health and wellbeing applications on positive physical activity behavior change particularly at the workplace i.e. after breakfast/lunch time (more details are described in the next section).

\section{SYSTEM DESIGN}

We designed a health and wellbeing application iGO to assist working people/employee in promoting their physical activities like exercise and walking. iGO is a simple persuasive health and wellbeing application that lets users to promote their physical activity and lets them to record whether they choose physical activity by promoting self-determination task and letting users to save their selections. Users use the application every time after the breakfast and lunch at workplace.

For iGO design, SDT sequence (autonomy support psychological needs - extrinsic motivation) for health behavioral change was adopted and integrated. Human easily feel lazy to do things willingly but may be tricked by external triggers. For example, text messaging reminders had been used for the diabetic patients to improve their self-efficacy to glycemic control [36]. Thus, a reminder i.e. alarm/vibration is recommended to be used in our application. The procedure of the iGO can be described as: an alarm/vibration reminder goes on just after breakfast recess, and the application then shows an option whether users had the breakfast or not by click "yes" or "no". If users did not have their breakfast and clicked "no" then alarm will appear again after 10 minutes and will ask users to choose for his/her option. If users click "yes", then it will ask users to choose "physical activity with others" or "physical activity by own". When user chooses the button "physical activity with others" time starts to count for 10 minutes and the user receives 2 points $(5$ minutes $=1$ point $)$ if he/she does exercise/walking. After completing the session "physical activity with others", the system will ask user to press the button "physical activity alone". When users choose the button "physical activity alone" time starts to count for 10 minutes and users receive 2 points $(5$ minutes $=1$ point $)$ if he/she does body movement. Points get deducted if choose "no physical activity" button. Similarly, an alarm/vibration goes on just after lunch break and follows the above procedure. At the end of the day, a social interactive display i.e. a result dashboard appears on the application to show the score of individual user. Users receive a personal image in accordance with their points, e.g. highest points user will get a nice portrait image whether other will get portrait image with a fatty face.

From user's perspective, designing application should mainly focus application operation, for example, what are its functions and how are they demonstrated and helped to users. Therefore, iGO application aimed to collect data and represent those after analyzing so that users receive useful information that make users more interested in using the application [37]. User data to record their point earnings was stored in a secured database. Earned points after physical activity were shown on the result dashboard and on the user's individual profile.
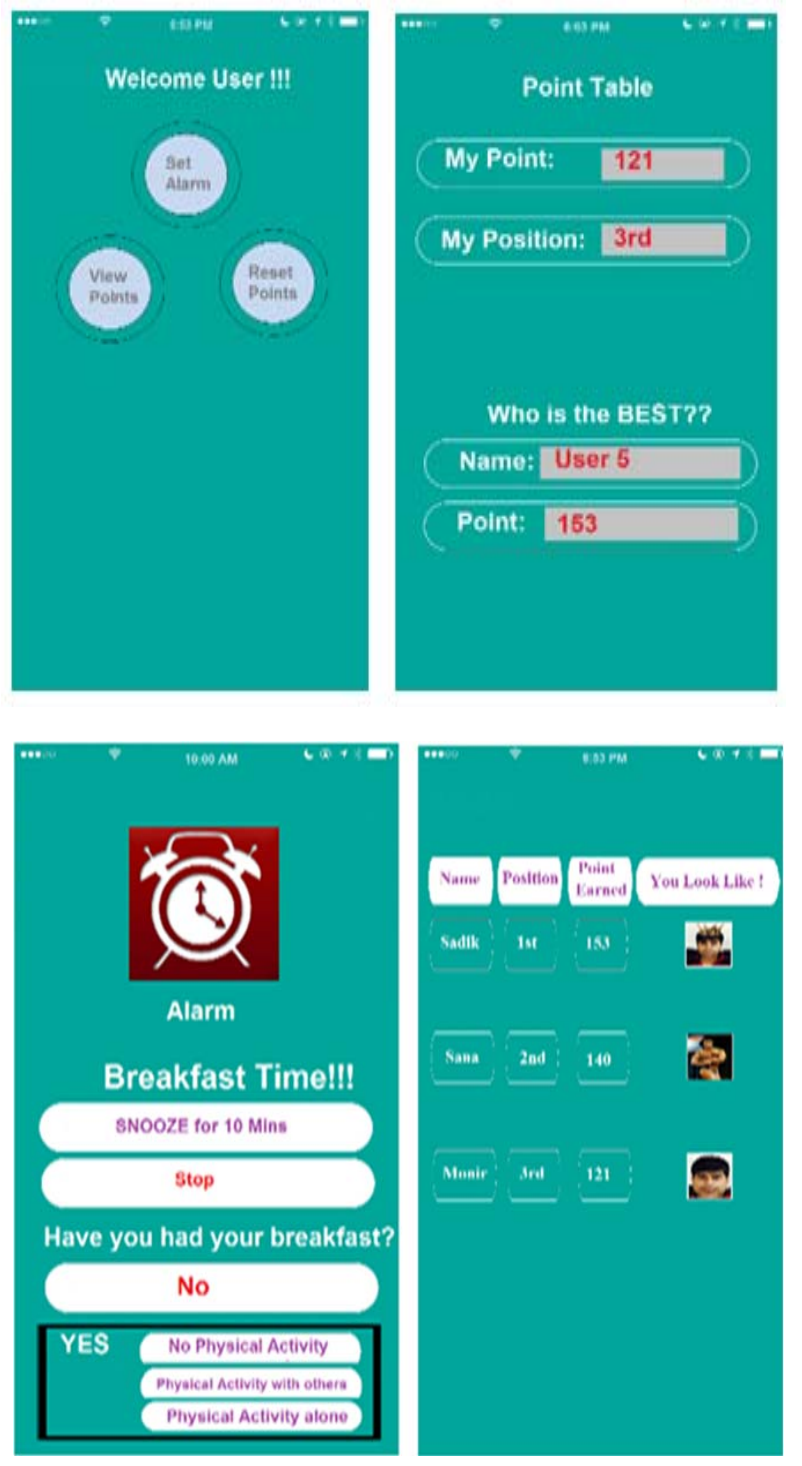

Fig. 2. Screen shots of iGO application

Fig. 2 represents screen shots of iGO application. The main procedure in designing the application is summarized below. Users manage our application e.g. sending alarm/vibration. Users have option to use/skip the application by pressing the "yes" or "no" button when asking whether he/she had breakfast/lunch. Users express their views and able to choose their options and choices through the application and thus, the application prompts to autonomous support. Clicking the button "physical activity with others" indicates user's expression of the psychological need relatedness as it urges to feel associated to other. On the other hand, clicking the button "physical activity alone" indicates user's expression 
of the psychological need autonomy as it "refers to being the perceived origin or source of one's own behavior," [23]. Social interactive display, i.e. a result dashboard appears on the application, which expresses the psychological need competence "as feeling effective in one's ongoing interactions with the social environment and experiencing opportunities to exercise and express one's capabilities". In addition, scored points with added personal goodwill image indicate rewards for users which are the extrinsic motivation. Therefore, our effort to integrate the SDT sequence with our application has been fulfilled. In the next section, we describe of how we tested the usability of the prototype.

\section{PROTOTYPE EVALUATION}

The over-all usability of the designed prototype was tested before we implemented our full application. Initially, test users were selected for a week-long study and we aimed to conduct our main evaluation study later (more details are described later). Test users used the application before the breakfast and lunch recess at the workplace in an office environment. The aim of the application test was to evaluate whether the application was friendly to use, and whether the users practiced the application after the breakfast/lunch for physical activity purpose for example choose button "yes" to go for physical activity. The prototype was installed in an android mobile-friendly web application and user data was stored on the database and the earned points were calculated accordingly.

For testing, 26 participants were recruited; $\mathrm{N}=26,10$ females and 16 males. All participants were between 25 and 41years of age healthy adults and were employed persons and had an interest in doing physical activity. Prior to the start of the test, an informed consent form was provided to the participants outlining the research study and expectation from them. A brief introduction was provided to the participants, explaining that it would motivate them to increase physical activity towards better productivity. Participants used our application for one week at their office environment. Postquestionnaires confirmed that participants were interested to use the application. 15 participants were interviewed and asked to describe their experience in using the iGO application. Participants were selected based on their availability and interest to be interviewed. While using iGO application, most participants felt that the iGO application really convinced them:"Before using the app, I was feeling lazy but after using the app, I felt amazing", "The apps is reminding me at least to do some physical things which is great", "I felt like losing weight which is great!", "Walking after meal does not make me feel to have extra weight on my body". To evaluate the usability, participants were asked to describe how hard or easy they felt in using the iGO application. Most participants reported the application as satisfactory and user-oriented; "user friendly, works as personal assistant", "very easy and convenient", "pretty good", "simple, easy to use", "good thinking app". However, some users complained that the application was compatible only with android operating system as they were using the iOS operating system.
The report from the application usability showed positive impacts in terms of using the iGO application. The participants' overall experiences surrounding the iGO application were positive.

Our current study is a work-in-progress paper. Initially, we developed iGO by adopting psychological constructs using the SDT theory. In our next study, we shall conduct the main evaluation study. This should be done as at least one month pilot study on the users using the application at their office environment. Two different health and wellbeing applications will be facilitated to users and their effects will be compared. Changes in work productivity will be followed. The first one remains iGO and the other one would be an ordinary health and wellbeing application that does not adopt theoretical constructs. Users shall be randomly assigned to practice health and wellbeing applications in two different conditions: experimental and control. The future study compares the new designed health and wellbeing application and the ordinary health and wellbeing application to access efficacy of our newly made health and wellbeing application.

\section{CONCLUSION}

Initially, our application is designed to improve user's interest to do exercise/walking to increase physical activity that lead to better working hour productivity. We implemented and tested our iGO, a persuasive health and wellbeing application that is designed to promote physical activity at the workplace, to increase the work efficiency and offer extrinsic rewards to motivate and feel good of self-determined motivation. We have been able to use theoretical construct SDT to promote physical activity methods for our application. It is the first application to promote physical activity at workplace after meal i.e. breakfast/lunch integrated by SDT. Overall, our designed application was user friendly and brought positive impacts in promoting physical activity. We also believe our further study shall gauge the effectiveness of our application to promote physical activity and improve work productivity.

\section{ACKNOWLEDGMENT}

We would like to give special thanks to the participants who helped us with the prototype evaluation.

\section{REFERENCES}

[1] ESSA (2012). Physical Activity in the Wrokplace: A Guide[Online] Availablefrom:<http:/www.usc.edu.au/media/3089/Physicalactivityinthe workplaceaguide.pdf $>$ [Accessed on 02.09.2016]

[2] World Health Organization (2002). Physical inactivity a leading cause of disease and disability, warns WHO [Online] Available from: $<$ http:www.who.int/mediacentre/news/releases/release23/en/> [Accessed on 07.09.2016]

[3] World Health Organization (2016). Global Activity on Diet, Physical activity and health[Online] Available from: $<$ http:www.who.int/mediacentre/news/releases/release23/en/> [Accessed on 07.09.2016]

[4] Eurobarometer (2010).Sport and Physical Activity. Special Eurobarometer, 334/Wave 72.3

[5] Prevalence of Regular Physical Activity Among Adults - United States, 2001 and 2005. MMWR.2007;56, pp.1209-1212 
[6] Biolo, B. Ciocchi, B. Stulle, M. Piccoli, A. Lorenzon, S. Dal Mas, V. Barazzoni, R. Zanetti, M. and Guernieri, G. (2005). "Metabolic consequences of physical inactivity." Journal of Renal nutrition 15(1), pp.49-53

[7] Roberts, C. K. and Laye, M. J. (2012). Lack of Exercise Is a Major Cause of Chronic Diseases. Comprehensive Physiology. 2, pp.11431211

[8] Kujala, M. (2009). Evidence on the effects of exercise therapy in the treatment of chronic disease. British Journal of Sports Medicine, 43, pp.550-555.

[9] Warburton, D.E.R., Nicol, C.W. and Bredin, S.S.D. (2006). Health Benefits of physical activity: the evidence. Canadian Medical Association Journal, 174(6), pp.801-809

[10] Atlantis, E., Barnes, E.H. and Ball, K. (2007). Weight status and perception barriers to healthy physical activity and diet beahviour. International Journal of Obesity, 10, pp.1-10.

[11] World Health Organization (WHO) report (2001). Workers health Global Plan of Action, Sixtieth World Health Assembly,

[12] Torning, K. and Kukkonen, H., (2009). Persuasive system design: state of the art and future directions. Proceedings of the 4th International Conference onPersuasive Technology, Springer, 30

[13] Brown, B., Chetty, G., and Harmon, E., (2012), A system for students to monitor diet and exercise. Proceedings of CHI, pp.1807-1812.

[14] Consolvo, S., Everitt, K, Smith, I I, and Landay, JA., (2006). Design requirements for technologies that encourage physical activity. Proceedings of CHI 2006, ACM Press, pp.457-466

[15] Baumer, E. P. S., Katz, S. J., Freeman, J. E., Adams, P., Gonzales, A. L., Pllak, J. P., Retelny, D., Niederdeppe, J., Olson, C. M., and Gay, G. K, (2012). Prescriptive Persuasion and Open-Ended Social Awareness: Expanding the Design Space of Mobile Health. Proceedings of CHI 2012, ACM Press, pp.476-484.

[16] Conroy, D., Yang, C. and Maher, J. (2014). Behaviour change techniques in top ranked mobile apps for physical activity, Am J Prev Med, 46(6), pp.649-52

[17] Croce. R., and Horvat, M. (1992). Effects of reinforcement based exercise on fitness and work productivity in adults with mental distraction. Adapted Physical Activity Quarterly, 9, pp.148-178.

[18] Middleweerd, A., Mollee J S, Wal, C N., Brug, J and Velde, S J. (2013). Apps to promote physical activity among adults: a review and content analysis,International Journal of Behavioral Nutrition and Physical Activity, 11(97).

[19] Cowan, L., Van. W. S., Brown, B., Hedin, R., Seino-Stephan, Y., Hall, P. and West, J. (2013), Apps of steel: are exercise apps providing consumers with realistic expectations?: a content analysis of exercise apps for presence of behavior change theory. Health Educ Behav, 40, pp.133-139.

[20] West, J., Hall, P., Hanson, C., Barnes, M., Giraud-Carrier, C. and Barrett J. (2012). There's an app for that: content analysis of paid health and fitness apps. J Med Internet Res, 14:e72

[21] Breton, E., Fuemmeler, B. and Abroms, L. (2011). Weight loss-there is an app for that! But does it adhere to evidence-informed practices?" Transl Behav Med, 1, pp.523-529

[22] Deci, E. L., and Ryan, R. M. (2002). Handbook of selfdeterminationresearch. Rochester, NY: University of Rochester Press
[23] Wilson, P. M., Mack, D. E., and Grattan, K. P. (2008). Understanding motivation for exercise: a self-determination theory perspective. Canadian Psychology, 49, pp.250-256

[24] Deci, E. L., and Ryan, R. M. (2000). The "what" and "why" of goal pursuits: Human needs and the self-determination of behavior. Psychological Inquiry, 11, pp.227-268. doi:10.1207/S15327965PLI1104_01

[25] Barbeau, A., Sweet, S. N., and Fortier, M. (2009). A path-analytic model of self-determination theory in a physical activity context. Journal ofApplied Biobehavioral Research, 14, pp.103-118. doi:10.1111/j.17519861.2009 .00043

[26] Russell, K. L., and Bray, S. R. (2009). Self-determined motivation predicts independent, home-based exercise following cardiac rehabilitation. Rehabilitation Psychology, 54, pp.150-156. doi: $10.1037 / \mathrm{a} 0015595$

[27] Fortier, M. S., Sweet, S. N., O’Sullivan, T. L., and Williams, G. C. (2007). A self-determination process model of physical activity adoption in the context of a randomized controlled trial. Psychology of Sport and Exercise, 8, 741-757. doi:10.1016/j.psychsport.2006.10.006

[28] Ryan, R. M., and Deci, E. L. (2008). A self-determination approach to psychotherapy: The motivational basis for effective change. Canadian Psychology, 49, pp.186-193.

[29] Kasser, T. andRyan, R. M. (1996). Further examining the American dream: Differential correlates of intrinsic and extrinsic goals', Personality and Social Psychology Bulletin 22, pp. 280-287.

[30] Ryan, R. M., Patrick, H., Deci, E. L, and Geoffrey C. Williams, G. C. (2008).Facilitating health behaviour change and its maintenance: Interventions based on Self-Determination Theory, European Health psychologist, 10

[31] Teixeira, P., Carraca, E, Markland, D, Silva, M, and Ryan, R. (2012). Exercise, physical activity, and self-determination theory: a systematic review. International Journal of Behavioral Nutrition and Physical Activity, 9

[32] Philip M. Wilson, Diane E. Mack, and Kimberly P. Grattan (2008). Understanding Motivation for Exercise: A Self-Determination, Theory Perspective, Canadian Psychology, Canadian Psychological Association, 49(3), pp. 250-256

[33] NWHU report (2014). Workplace Health. [Online] Available from: $<$ https:www.nwhu.on.ca/ourservices/WorkplaceHealth/Documents/PA\% 20Resource\%20Kit\%20for\%20Workplaces\%20May\%202014.pdf $>$ [Acc essed on 06.09.2019]

[34] David R. Lubans, Jordan J. Smith, Geoff Skinner and Philip J. Morgan (2014). Development and implementation of a smartphone application to promote physical activity and reduce screen-time in adolescent boys, Public Health Education and Promotion, 2(42)

[35] Yasuyo, H. and Seika, Y. (2011). Walking just after a meal seems to be more effective for weight loss than waiting for one hour to walk after a meal, International Journal of General Medicine, 4, pp.447-45

[36] Franklin, V. L., Waller, A., Pagliari, C. and Greene, SA. (2006). A randomized controlled trial of Sweet Talk, a text-messaging system to support young people with diabetes, 23(12), pp. 1332-1338

[37] Tuovinen, L., Ahola, R., Kangas, M., Korpelainen, R., Siirtola, P., Luoto, T., Pyky, R., Röning, J. and Jämsä, T. (2016). Software Design Principles for Digital Behavior Change Interventions - Lessons Learned from the MOPO Study.In Proceedings of the 9th International Joint Conference on Biomedical Engineering Systems and Technologies, 5 